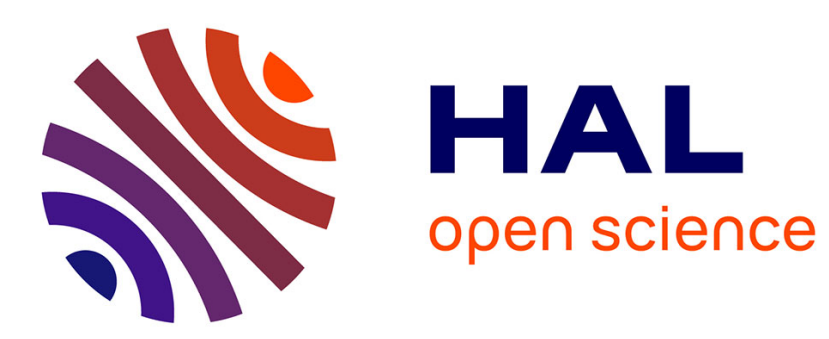

\title{
Planning and Scheduling for Optimizing Communication in Smart Grids
}

Miroslav Kadlec, Barbora Buhnova, Tomas Pitner

\section{To cite this version:}

Miroslav Kadlec, Barbora Buhnova, Tomas Pitner. Planning and Scheduling for Optimizing Communication in Smart Grids. 12th International Symposium on Environmental Software Systems (ISESS), May 2017, Zadar, Croatia. pp.446-456, 10.1007/978-3-319-89935-0_37 . hal-01852628

\section{HAL Id: hal-01852628 \\ https://hal.inria.fr/hal-01852628}

Submitted on 2 Aug 2018

HAL is a multi-disciplinary open access archive for the deposit and dissemination of scientific research documents, whether they are published or not. The documents may come from teaching and research institutions in France or abroad, or from public or private research centers.
L'archive ouverte pluridisciplinaire HAL, est destinée au dépôt et à la diffusion de documents scientifiques de niveau recherche, publiés ou non, émanant des établissements d'enseignement et de recherche français ou étrangers, des laboratoires publics ou privés.

\section{(c)(1)}

Distributed under a Creative Commons Attribution| 4.0 International License 


\title{
Planning and Scheduling for Optimizing Communication in Smart Grids
}

\author{
Miroslav Kadlec ${ }^{1}$, Barbora Buhnovaํㅜㄹ and Tomas Pitner ${ }^{1}$ \\ ${ }^{1}$ Faculty of Informatics, Masaryk University, \\ Brno, Czech Republic \\ Email: \{miroslav.kadlec, buhnova, tomp\}@mail.muni.cz
}

\begin{abstract}
Smart grid is a concept defining future electricity distribution network with the purpose of improving its reliability, efficiency and reducing its ecological impact. To achieve that, massive volumes of data need to be sensed and transmitted between the elements of the grid. A robust and efficient communication infrastructure is thus as essential part of smart grid. Some of the data transmissions are not time-critical, providing an opportunity to improve the communication network performance. In this paper, we present a new approach to optimize smart grid communications through time-based scheduling. Additionally, we provide a review of communication technologies in smart grids and published approaches to avoid congestions and transmission failures.
\end{abstract}

Keywords: Smart Grids · Communication · Scheduling · Optimization

\section{Introduction}

The traditional electricity distribution infrastructures were developed during the first half of the $20^{\text {th }}$ century and nowadays, they are undergoing complex transformation to fully satisfy the needs of modern society. The electricity consumption has doubled in the past 20 years and it is supposed to keep rising at least the same speed. Since the majority of electricity generation relies on coal, oil and gas resources, the growth in power usage brings more and more significant environmental problem. Additionally, more aspects of modern life are dependent on the electricity supply. Though, incorporating renewable resources based generators (e. g. photovoltaic power plants and wind turbines) as well as making the whole infrastructure more monitorable and controllable become an important strategic act to preserve power grid sustainability.

The need to employ more power generators based on renewable sources brings several challenges to be solved. First, both wind and solar power plants provide rather unstable electricity production varying with current weather that is difficult to be predicted, but may be estimated from short term weather forecast. Nowadays, end electricity users are also encouraged to install their own small power sources in order to save money lowering the electricity consumption and potentially send electricity back to the network in cases of overproduction. Since storing energy or transmitting it over long distances causes significant losses, the task of keeping the power grid capable of maintaining these changes is nontrivial.

The concept of smart grid represents a complex approach to improve current power distribution infrastructure in order to provide application such as demand response, use 
of distributed energy resources (DER) or real-time state monitoring [1]. The overall goals are to lower energy losses and to make the electricity network more reliable, efficient and environmental-friendly. In order to achieve the previously mentioned applications, smart metering and remotely controllable devices, communication infrastructure and computational systems for data collection and decision making are supposed to be added to the existing power distribution network.

To make the grid state analysis and decision making as relevant as possible, a large amount of up-to date complete data will be needed. Typically, the data will be generated in metering/monitoring points all over the network and then collected in central offices and, conversely, control commands will be sent from central offices to elements of the grid. Hence, a bidirectional, robust and reliable communication network is a critical part of smart grid. Due to its complexity, expenses associated with deploying a communication infrastructure of this scale are supposed to be high and strongly dependent on concrete technology used.

The communication requests are expected to have variable data sizes and requirements on latency and reliability of delivery. Since some of the data transfers are not time critical, the communication may be planned using proper scheduling techniques, request importance evaluation, real time adaptation and predictions of network traffic load and message sizes in order to balance traffic load and to prevent and manage communication network overloads. Thus, a given communication infrastructure will be capable to maintain bigger number of communication requests and to provide most relevant data possible at the time, so a better trade-off between quality and price of the communication infrastructure may be earned. To schedule the messages efficiently is a new optimization problem with many aspects specific to smart grid environment.

In this paper, we propose new approach to optimize smart grid communications through time-based scheduling. As the first step, an analysis of smart grid communication was conducted, based on existing literature as well as our practical experience from the smart grid design and implementation projects that we actively contribute to within the context of the Czech Republic. Then, the optimization problem is identified along with its inputs as well as techniques to find the solution are outlined.

The rest of the paper is organized as follows. In the Section 2, messages exchanged between smart grid elements are summarized with emphasis to their communication requirements. In the Section 3, communication technologies usable for smart grids with their specifics and challenges are listed. In the Section 4, the opportunity to use a scheduling algorithm in order to optimize traffic load is described and design of a potential solution is outlined and the specifics of given optimization problems are described.

\section{Communication in Smart Grids}

As long as smart grids are being developed as enhancement of current electricity distribution networks rather than brand-new infrastructures, the concrete implementations vary through different countries according to the existing power grids specifics. On the other hand, the overall structure, high-level applications and other general characteristics remain the same. In order to provide a standardized model of 
smart grid basic structure, SGAM (Smart Grid Architecture Model) framework has been presented in [2]. Although the model omits technical and implementation details, it provides a good insight into the organizational structure and defines several levels of abstraction the smart grids may be looked on, Fig 1. Along the "interoperability" axis, several layers are listed, where each one is supported by layers located below. For example, the function layer describes functions and services provided by the smart grid in order to fulfill business requirements and policies. Similarly, the information layer summarizes all the data needed by the functions.

According to the SGAM framework, communication infrastructure serves as the support element for all the upper layers. Indeed, having up to date data transmitted by communication infrastructure is necessary for the data-analysis and decision-making systems incorporated in smart grids [1][3]. Thus, deploying a robust communication infrastructure capable of satisfying smart grid requirements is one of major tasks in smart grid development [4][5].

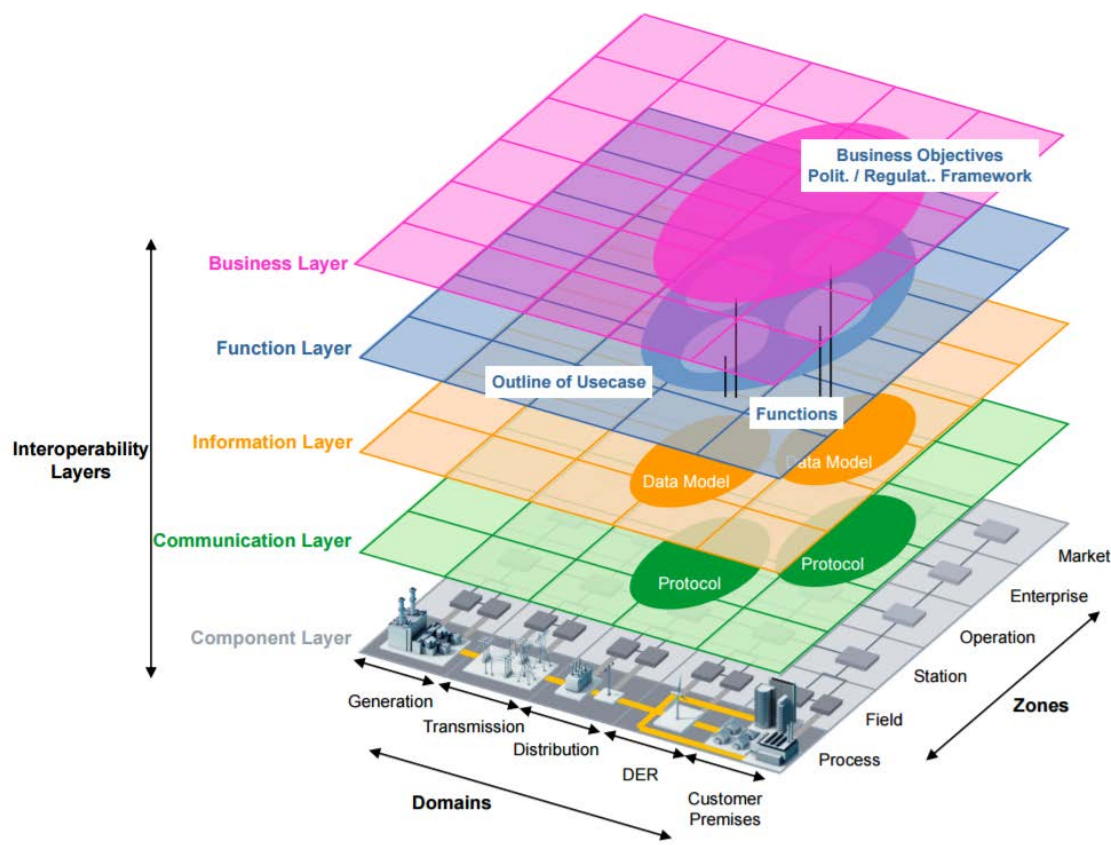

Fig. 1. Smart Grid Architecture model [2].

According to [6][7], the communication network itself may be divided into three domains according to the coverage range and involved devices:

- Home area network (HAN), that realizes communication between the smart meter and other household appliances, for example local power generators, controllable consuming devices, electric cars and other devices equipped by communication interface.

- Neighborhood area network (NAN) interconnects smart meters and data concetrators, that collect metering data and send them to central systems. Complementarily, control commands coming from central systems are passed to smart meters. 
- Wide area network (WAN) cover data exchange between data concentrators and the central systems, where the collected data is analyzed and potentially reacted to.

A graphical representation of described architecture is in Fig. 2, showing HAN, NAN and WAN with typical communicating elements. Such a multi-layered model is expected to be used since the smart grid communication network is needed to cover entire region with the intention to connect a large set of nodes [8].

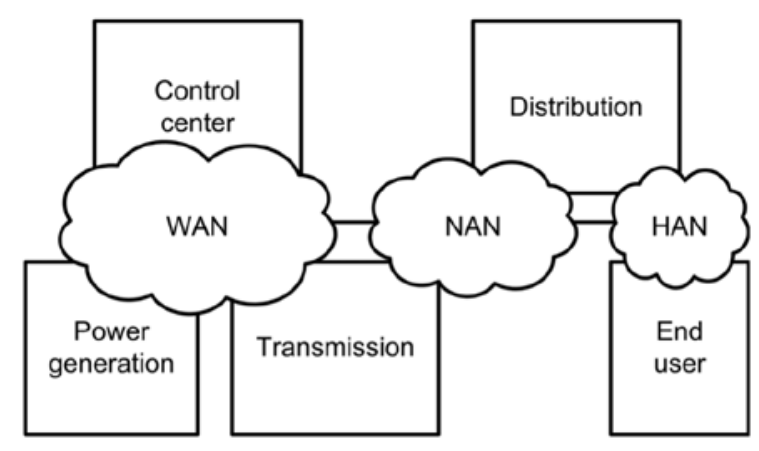

Fig. 2. Multilayer structure of the smart grid communication network [8].

\subsection{Communication Requirements}

Several papers have been published aiming to describe communication requirements of smart grids. The general objective of making the power distribution network more efficient, reliable and inteligent is expected to be achieved through implementing certain smart grid applications. The following requirements on the smart grid are the most commonly presented in existing studies [3][9][10][11]:

- Automatic metering reading (AMR) refers to a technique that uses a communication infrastructure to automatically collect metering data, events and alarms from the smart meters. Typical AMR communication scenarios are meter readings (both on-demand and previously scheduled readings), meter events and alarms (e. g. health events, unauthorized meter access), grid events detected by meters (temporary power outages) and others. AMI itself may be viewed as the most basic and simple application that serves as a platform supporting other applications [3][9].

- Wide area situational awareness will monitor and manage all the components of the electric power system. Through predicting and modifying behavior of smart grid elements potential problems in the infrastructure may be detected or avoided [3].

- Demand response and consumer efficiency aims at balancing the overall electricity consumption by encouraging customers to cut their usage during peak times. In order to achieve this behavior, dynamic electricity pricing will enable the consumers to lower their expenses by postponing some 
consumption, that is not time critical (e. g. electric water heater or air conditioning) to off-peak times [3][9].

- Distributed energy resources uses load prediction and balance techniques in order to optimize integration of big number of small electricity generators based on renewable sources [11].

- Grid-to-vehicle, vehicle-to-grid (G2V, V2G) tends to use integrated accumulators of electric and hybrid vehicles as temporary electricity sources in order to overcome short-term consumption peak times [12].

Although, the overall smart grid system is lacking widely accepted standards, for particular smart grid applications, standards and protocols have been published. In [13], major smart grid standards are listed and described, e. g. ANSI C12.1, ANSI C12.19 (Advanced metering infrastructure), OpenADR (Dynamic pricing) and SAE J2836 (Electric vehicles communication).

With the purpose of defining technical requirements on smart grid communication network, research has been conducted to analyze general business communication requirements. According to [14][15], reliability, latency, security and interoperability are the key characteristics of communication network in the context of smart grids requirements.

Concrete messages exchanged within smart grids are supposed to have various requirements depending on related smart grid application. In [16], utility operation practices, planned implementations and experience from other research groups have been analyzed in order to enumerate particular communication events with its estimated packet sizes, frequency, and delay objective. In the presented results, there are considerable differences between separate events requirements - while interval data reading is stated to have 480 Bytes data size, it is not considered to be delay time critical; on the other hand, meter remote disconnect requests size is only 20 Bytes, but the delay objective is 2 seconds.

Even more exhaustive summary has been published in [17], presenting typical data size, sampling frequency, maximum latency and desired reliability for various NAN and WAN applications. Twelve groups of smart grid operations have been investigated, for example:

- Advanced metering infrastructure applications including on-demand, scheduled and bulk metering data reads

- $\quad$ Pricing applications, e. g. TOU (time-of-use) commands, real-time pricing and critical peak pricing

- $\quad$ Electric service prepayment

- Demand response communication

According to the results presented, there is a big variability of both data size and latency requirements of separate operations.

\subsection{Communication Technologies}

Various communication technologies have been considered for smart grids including both wire-line and wireless solutions with respect to their abilities to maintain the requirements listed in the previous section and additionally to other aspects like coverage range and deployment costs, comparing their suitability for smart grids needs. 
Wireline technologies represent one option to build up communication network separate from the power distribution infrastructure itself. Construction of such a network would require extra investments; on the other hand, it is capable of providing higher data transmission rates and not shared with other application [18]. Some national-scale wireline networks have already been deployed for other purposes, e. g. cable television or telecommunications. Various transmission media may be possibly used.

Fiber-optic networks provide both the highest data rate (for SONET/SDH architecture, the maximum theoretical rate is $10 \mathrm{Gbps}$, for VDM technology up to 40 Gbps) and large coverage range (up to $100 \mathrm{~km}$ ) [17].

DSL (Digital Subscriber Line) technology relies ordinary telephone lines. ADSL (Asynchronous DSL) is capable of max theoretical data rate 1-8 Mbps downstream with coverage up to $5 \mathrm{~km}$. High-speed versions of DSL (HDSL, VDSL) provide higher data rates, but smaller coverage range [19]. Although, DSL architecture is already deployed for many premises that would lower the initial installation expenses, it may not be suitable for smart grid applications due to possible down time problems and low reliability especially for more distant customers [17].

Coaxial cable transmission network uses cable television infrastructure. For highspeed data transfer over existing hybrid fiber-coaxial network, DOCSIS (Data Over Cable Service Interface Specification) may be used. This technology provides $172 \mathrm{Mbps}$ maximum data rate and up to $28 \mathrm{~km}$ coverage range [17].

Ethernet is a technology often used to build local networks in houses and workplaces providing data rates between $10 \mathrm{Mbps}$ and $10 \mathrm{Gbps}$ [14]. Unlike DSL and Coaxial solutions, there is no Ethernet based large-scale network currently deployed.

Powerline Communication (PLC) may be viewed as a special case of wireline technology, but has several unique characteristics. PLC is realized through power distribution network. To transmit both the power and data, electric signal modulation is used. As long as the theoretical data rate is about hundreds of kbps and the transmission channel is rather noisy, PLC is not as effective as e. g. DSL [17]. However, it is not shared with other applications, that generate external traffic and though, cause inpredictable and unbalanced load.

Wireless communication technologies are also often inspected for the use in smart grids, mainly due to the fact, no wires need to be installed. Concrete wireless technologies may represent appropriate solutions for NANs or WANs.

Wireless Local Area Network (WLAN) is high speed communication technology often used for wireless Internet access commonly known as WiFi. It provides reliable secure communication with data rate up to 2-600 Mbps (depending on standard), but its maximum range is lower than $100 \mathrm{~m}$ and the cost and power consumption are higher making WLAN less suitable for massive smart grid use [17].

ZigBee operates on unlicensed bands (shared with WLAN) and its maximum theoretical data rate is $250 \mathrm{kbps}$. Due to its low speed, short range coverage (up to 100 $\mathrm{m})$, ZigBee may be suitable for in-home applications [14][17].

Cellular networks, e. g. GSM, GPRS, LTE, have the potential to enable wide area wireless smart metering deployment. The technology is based on a large number of transcievers dividing the covered area into communication cells. Communication devices connect to them do send and receive messages. In [20], GSM is considered a 
possible solution for smart grid communication since it is already deployed in most of inhabited areas. On the other hand, performance reduction during peak traffic and the problems connected to maintaining QoS over noisy channel.

Along with comparing technical parameters, Cost Benefit Analysis is an important procedure to assess the suitability of a particular technology for smart grid solutions. In [21], a comprehensive assessment framework of smart grid projects is proposed with purpose to provide guidance and advice for conducting cost-benefit analysis. According to the presented research, for monetizing possible benefits related to the smart metering roll-out, following aspects are critical (not exhaustive):

- Hardware cost including investments in communication infrastructure installation

- Data transfer costs (in GPRS, Radio Communications, ...)

- Communication success rate

- Number of smart metering installed

Hence, achieving a good trade-off between communication network costs and its robustness and capability is an important step in order to make the smart grid deployment effective and beneficial. Both PLC and GPRS are mentioned as technologies potentially usable for smart grid. Additionally, a comparison is presented, regarding PLC as more suitable for "concentrated" deployments (bigger number of clients concentrated in a limited area e. g. an entire city) and more initial-costs intensive, while GPRS is stated to work well in "scattered" deployments (e. g. only clients with higher consumption in each network).

\section{Optimizing Smart Grid Communication}

In recent years, several papers have been published with the purpose to identify opportunities to optimize smart grid communication, describing possible approaches and solutions to the presented optimization problem.

\subsection{Communication infrastructure design}

Proper technology choice and topology design is the first step to build a sustainable communication infrastructure. In [16], an approach is presented to estimate communication profiles for selected smart grid application. Then, the capacity utilization ratio is applied with respect to the traffic described while considering two scenarios (Blue Sky Day and Storm Day Scenario). As an output of this analysis, both the regional and WAN capacity requirements are derived. In [22], the scalability of three communication architectures for advanced metering infrastructure (including i. e. AMR) is inspected. Accumulated bandwidth-distance product (ABDP) is defined as a performance metric. For each architecture, an optimization problem is defined in order to obtain the solution for minimizing the total costs considering both the ABDP and the cost of meter data management system. Additionally, the scalability of the total expenses for the centralized and distributed infrastructure is analyzed and compared, while the distributed architecture is said to have significantly lower total cost [22]. 


\subsection{Congestion avoidance}

The problem of potential congestion caused by huge number of data samples has already been addressed in literature. TCP based networks may possibly be overloaded due to excessive packet retransmission in case of communication peak times. To overcome this problem, so-called transport aggregator operating on communication transport level is presented in [23]. The aggregator node is added between the data sources and the data collection server in order to split the TCP connections between the mentioned elements. Its task is to collect data from sources (e. g. smart meters) and to forward to the central server while adjusting the transmission rate and achieving congestion control.

Another investigated method is to identify the most salient data to be communicated [18]. Through Singular Value Decomposition (SVD) analysis, the authors have determined, that typical grid admittance matrices have only small number of strong components. SVD sparsity proved to be able to find out, what parts of the system are strongly coupled [18].

In [24], an intelligent traffic volume reduction of the communication flows is presented. The authors note that beside of "essential" data that must reach the target, there is a "non-essential" part. An assumption is made, that due to limited bandwidth, the communication infrastructure is not capable to transmit all the data successfully. Quality-aware reduction of the non-essential data component is used to utilize the overall revenue of communication flows processed.

In [25], a systematic priority-based approach is described to model and optimize communication in sensor networks over cognitive radio (CR) infrastructure. The proposed algorithm performed well in simulations, however is tightly connected to the concrete technology used.

To the best of authors knowledge, time-based scheduling of data transmissions in order to optimize communications in smart grids has not been investigated exhaustively. We have addressed this problem in cooperation with the major electricity distribution company in the Czech Republic. An AMR (Automated Meter Reading) pilot project is being executed within particular regions providing an opportunity to explore ways to optimize communications. In the current setting, all the data collection are executed at fixed times that were assigned considering neither the network specifics nor the current state if the infrastructure. As long as particular communication requests have various requirements in scope of delivery time and message size, proper timebased scheduling may be used to prevent the network from congestion and potential message interferences. In the first part of the research described in this paper, we focus on WAN communication scheduling, thus the messages in following text are the ones transmitted between a central management system and Distribution Transformer Stations (DTS).

\subsection{Time-based communication scheduling problem}

We have analyzed the current AMR environment identifying the following groups of characteristics important for implementing an appropriate communication scheduler.

First, we have inspected the specifics of cellular network that is going to be used in the scope of the Czech Republic (and likely also in other European countries) for WAN communication. The cellular network consists of base transceiver station (BTS) 
distributed within the covered area. The following aspects have been considered crucial for the scheduling process:

- Sending big amount of data through a single BTS may lead to undesired interferences and potential overload of given transceiver.

- It is uncertain, which BTS a concrete element communicates through.

o Nevertheless, the probability that two DTS will communicate through the same BTS might be partially estimated according to their geographic location.

- The BTS capacity varies (due to external traffic, weather conditions). Estimation and detection of critical periods would help to avoid scheduling requests in such intervals.

Single DTS is typically unable to serve more simultaneous communication requests.

Additionally, we have determined, which attributes each communication request should be assigned with in order to describe its characteristics and requirements:

- target device,

- desired interval, when the given request needs to be processed, determined by start time and deadline.

- priority preference describing, when the request should be preferably processed (e. g. control commands should be scheduled as soon as possible; on the other hand, in some cases of metering data reading, it may be beneficial to schedule them later, providing data concentrator with extra time to collect more data),

- estimated data load and duration of the request.

Since neither the data describing communication network nor the requests characteristics (data load, processing time) are often available, collecting message sizes, failure rate and processing times will be an important part of the scheduling algorithm in order to build a sufficient knowledge base. Gathered information will be then used to enhance the accuracy of predicted values essential for proper scheduling.

We assume that not all the communication requests will be known during schedule creation. Though, the scheduling needs to be done on both a strategic and operational level. While the strategic-level scheduling algorithm of previously known requests (e. g. periodic meter readings) is not necessarily time critical (and may be able to analyze all the inputs mentioned), light-weight techniques need to be implemented to dynamically readjust scheduled messages in case of sudden latency-critical messages generation (e. g. ad-hoc meter readings, smart meter commands).

Finally, since the meter data readings represents a significant part of all data transmitted, the overall traffic may be reduced through identifying less critical data (e. g. periodic metering data of low-consumption places) and potentially omitting them in case of overload. 


\subsection{Future work}

Proposed analysis was conducted as part of a pilot project defined by Czech energy distribution company aiming to deploy AMI in particular areas. In the scope of the Czech Republic, the PLC is most probably to be used for NAN communication, while the WAN will mostly use cellular network (GPRS and LTE).

Future effort will aim at implementing systems for data collection, analysis and prediction in order to obtain the essential inputs described in the previous section and then, to design and implement a prototype of the proposed communication scheduler itself.

\section{Conclusion}

Smart grid is a complex approach to make currently existing power grids more robust, efficient and sustainable. Beside other applications, smart grids aim at balancing electricity consumption, incorporate renewable energy resources, monitor the whole system and avoid or recover from faults. To achieve that, a communication infrastructure is used to interconnect smart metering devices deployed in the area with central systems for data analysis, prediction and decision making.

To maintain huge amounts of data, a sufficient communication network needs to be deployed. Along with optimization techniques listed in sections 3.1, 3.2, time based messages scheduling is believed to be able to decrease the risks of network overloading and congestion. Hence, a better trade-off between capability and price of the communication infrastructure can be earned. In this paper, an approach to optimize smart grid communication through communication requests scheduling is presented along with identification of essential input data of the proposed scheduler. As a part of the work presented in this paper, an extensive review of the state of the art of technologies and existing literature resources have been performed, which became part of Sections 2 and 3, to underline the reasoning that follows these sections.

\section{$5 \quad$ References}

1. Saputro, N., Akaya, K. (2012). A survey of routing protocols for smart grid communications. National Institute of Standards and Technology, 11, (1).

2. Smart Grid Coordination Group: Smart Grid Reference Architecture. (2012). CEN-CENELEC-ETSI.

3. Gao, J., Xiao, Y., Liu, J., Liang, W., \& Chen, C. P. (2012). A survey of communication/networking in Smart Grids. Future Generation Computer Systems, 2, 391-404.

4. Madueno, G. C., Stefanovic, C., Popovski, P. (2013). How Many Smart Meters can be Deployed in a GSM cell? (pp. 1263-1268).

5. Kouhdaragh, V., Tarchi, D.,Vanelli-Coralli, A., Corazza, G. E. (2015). Smart meters density effects on the number of collectors in a Smart Grid. (pp. 476-481).

6. Yu, R., Zhang, Y., Gjessing, S., Yuen, C., Xie, S., Guizani, M. (2011). Cognitive radio based hierarchical communications infrastructure for smart grid. IEEE network, 5, 6-14.

7. Gungor, V. C, Bucella, C., Hancke, G. P. (2012). Advance Metering Infrastructure and DLMS/COSEM Standards for Smart Grid. 1(2). 
8. Bojkovic, Z., Bakmaz, B. (2012). Smart grid communications architecture: a survey and challenges. Proceedings of the 11th International conference on Applied Computer and Applied Computational Science, 1, 83-89.

9. Khan, R. H., \& Khan, J. Y. (2013). A comprehensive review of the application characteristics and traffic requirements of a smart grid communications network. Computer Networks, 57, 825-845.

10. Locke, G., \& Gallagher, P. D. (2010). NIST framework and roadmap for smart grid interoperability standards, release 1.0. 33 .

11. Brown, R. E. (2008). Impact of smart grid on distribution system design. Power and Energy Society General Meeting-Conversion and Delivery of Electrical Energy in the 21st Century, 1, 1-4.

12. Khan, R. H., \& Khan, J. Y. (2013). A comprehensive review of the application characteristics and traffic requirements of a smart grid communications network. Computer Networks, 3, 825-845.

13. Gungor, V. C., Sahin, D., Kocak, T., Ergut, S., Buccella, C., Cecati, C., \& Hancke, G. P. (2011). Smart grid technologies: communication technologies and standards. IEEE transactions on Industrial informatics, 7(4), 529-539.

14. Wang, W., Xu, Y., \& Khanna, M. (2011). A survey on the communication architectures in smart grid. Computer Networks, 15, 3604-3629.

15. Gungor, V. C., Sahin, D., Kocak, T., Ergut, S., Buccella, C., Cecati, C., \& Hancke, G. P. (2013). A survey on smart grid potential applications and communication requirements. IEEE Transactions on Industrial Informatics, 1, 28-42.

16. Luan, W., Sharp, D., \& Lancashire, S. (2010). Smart grid communication network capacity planning for power utilities.. IEEE PES T\&D, 1, 1-4.

17. Kuzlu, M., Pipattanasomporn, M., \& Rahman, S. (2014). Communication network requirements for major smart grid applications in HAN, NAN and WAN. Computer Networks, 67, 78-88.

18. Wang, Z., Scaglione, A., \& Thomas, R. J. (2010). Compressing electrical power grids. (pp. 13-18).

19. Habib, A., \& Saiedian, H. (2012). Channelized voice over digital subscriber line. Habib, A., \& Saiedian, H. (2002). Channelized voice over digital subscriber line. IEEE Communications Magazine, 40(10), 94-100.

20. Usman, A., \& Shami, S. H. (2013). Evolution of communication technologies for smart grid application. Renewable and Sustainable Energy Reviews, 19, 191-199.

21. Giordano, V., Onyeji, I., Fulli, G., Jimnez, M. S., \& Filiou, C. (2012). Guidelines for cost benefit analysis of smart metering deployment. JRC Scientific and Tech. Research.

22. Zhou, J., Hu, R. Q., \& Qian, Y. (2012). Scalable distributed communication architectures to support advanced metering infrastructure in smart grid. IEEE Transactions on Parallel and Distributed Systems, 23(9), 1632-1642.

23. Khalifa, T., Naik, K., Alsabaan, M., Nayak, A., \& Goel, N. (2010). Transport protocol for smart grid infrastructure. (pp. 320-325).

24. Allalouf, M., Gershinsky, G., Lewin-Eytan, L., \& Naor, J. (2011). Data-qualityaware volume reduction in smart grid network. (pp. 120-125).

25. Huang, J., Wang, H., Qian, Y., \& Wang, C. (2013). Priority-based traffic scheduling and utility optimization for cognitive radio communication infrastructurebased smart grid. IEEE Transactions on Smart Grid, 1, 78-86. 